\title{
Tridimensional spectroscopy of Seyfert Galaxy 3C 120
}

\author{
G. Kosugi ${ }^{1}$, H. Ohtani, M. Yoshida ${ }^{2}$ and K. Aoki \\ Department of Astronomy, Faculty of Science, Kyoto University, Kyoto \\ 606-01
}

\begin{abstract}
Tridimensional spectroscopy of the Seyfert 1 galaxy 3C 120 was carried out with Spectronebulagraph (SNG). We constructed a data cube which is free from atmospheric variations during the scans. We then separated the blended lines by deconvolving the line profiles, to obtain pure narrow-line images and narrow-line intensity ratio diagram.
\end{abstract}

\section{Observations and Data Reductions}

The observations were made with $1.88 \mathrm{~m}$ telescope at Okayama Astrophysical Observatory using SNG (Kosugi et al. 1994a). The object was scanned within $30^{\prime \prime}$ by the $4^{\prime} .5$ slit of the spectrograph in two directions which were orthogonal to each other. The data reductions were made with SNG data processing system developed on IRAF. ${ }^{3}$ The intensities of $2 \mathrm{D}$ spectra obtained were scaled so that the intensities at the overlapping points of two orthogonal spectra coincide at a selected wavelength. Thus, we constructed a data cube with no infection from atmospheric variations. In order to deconvolve the narrow-lines and measure the intensities, multi/single-Gaussian profiles were fitted to the spectra. We then reconstructed the pure narrow-line images (Fig. 1).

The details, more results and discussions will appear in a forthcoming paper (Kosugi et al. 1994b).

\section{Results and Discussions}

Emission line regions are well classified by two dimensional diagram of selected line ratios (Veilleux \& Osterbrock 1987; VO diagram hereafter). The reddening free narrow-line ratios [OIII] $5007 \AA / H \beta$ of the region shown in Fig. 1(d) are plotted as a function of $[\mathrm{NII}] 6583 \AA / \mathrm{H} \alpha$ (Fig. 2) . On Fig. 2, we overplotted the model curves calculated by photoionization code CLOUDY (Ferland 1991)

\footnotetext{
${ }^{1}$ Present Address: Department of Astronomical Science, The Graduate University for Advanced Studies, National Astronomical Observatory, Tokyo 181

${ }^{2}$ Present Address: Okayams Astrophysical Observatory, Okayama 719-02

IRAF is distributed by the National Optical Astronomy Observatories, which is operated by the Association of Universities for Research in Astronomy, Inc. (AURA) under cooperative agreement with the National Science Foundation, USA
} 


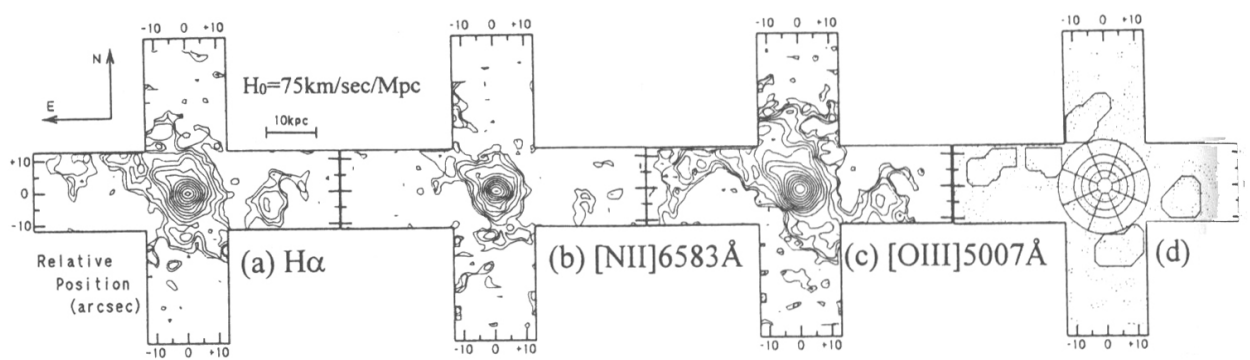

Figure 1. The pure narrow-line images. (a) Ha, (b) [NII]6583 $\AA$, (c) $[O I I I] 5007 \AA$ and $(d)$ integrated sections.

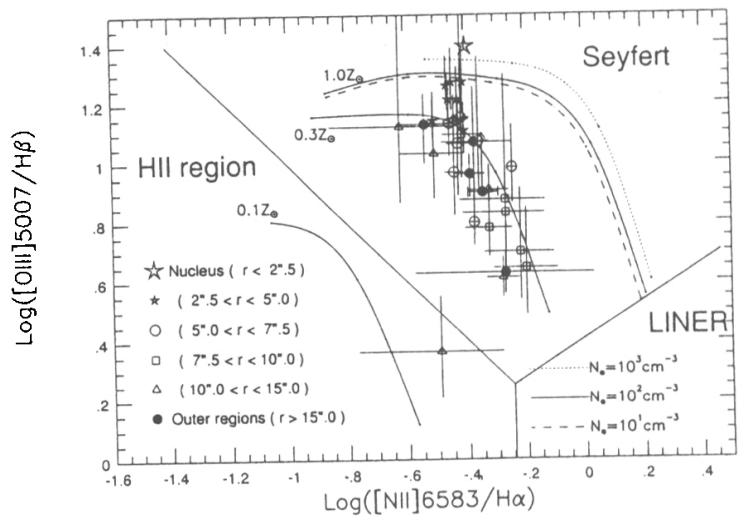

Figure 2. The VO diagram of $3 \mathrm{C} 120$. Each plot shows the line ratios of integrated spectra at the regions shown in Fig.1(d). The solid lines indicate the empirical boundaries between Seyfert, LINER and HII region. $N_{e}$ and $Z_{\odot}$ represent electron density and the solar abundance value, respectively. Ionization parameter $U$ varies along the curves

using the optimized model ionizing continuum. This curve is not so affected by the change of $N_{e}$. If the emission from HII region is contaminated, it's hard to explain the behaviors of the data points along the model curve. Then EELR is attributable mainly to the nuclear UV radiation, with varying the ionization parameter $U$. The regions outer $5^{\prime \prime} .0$ from the nucleus have roughly 0.3 times solar abundance, whereas the nuclear abundance is solar.

\section{References}

Ferland, G. J. 1991, OSU Internal Report 91-01

Kosugi, G., Ohtani, H., Sasaki, T., Shimizu, Y., Koyano, H., Yoshida, M., Baba, A., Aoki, K., \& Sasaki, M. 1994a, in preparation

Kosugi, G., Ohtani, H., Yoshida, A., \& Aoki, K. 1994b, in preparation

Veilleux, S., \& Osterbrock, D. E. 1987, ApJS, 63, 295 